INTERMATIONAL JOURNAL
PHARMACEUTICAL SCIENCES
RESEARCI
RESEI

Received on 15 August, 2017; received in revised form, 12 November, 2017; accepted, 17 November, 2017; published 01 June, 2018

\title{
FORMULATION AND EVALUATION OF METRONIDAZOLE EFFERVESCENT GRANULES
}

\section{A. Salomy Monica Diyya * and Noel Vinay Thomas}

Bharat Institute of Technology - Pharmacy, Rangareddy - 501510, Telangana, India.

\section{Keywords:}

Effervescence, Meltable binder, Effervescence cessation time, In-vitro drug release studies

Correspondence to Author:

\section{A. Salomy Monica Diyya}

Assistant Professor, Bharat Institute of Technology Pharmacy, Rangareddy - 501510, Telangana, India.

E-mail: monicadiyya@gmail.com

\begin{abstract}
Efferevescent granules are granular dosage form of drug in a dry mixture usually composed of effervescents like sodium bicarbonate, citric acid and tartaric acid. Effervescent granules when added to water, the acids and the base react to liberate $\mathrm{CO}_{2}$, resulting in effervescence. A combination of tartaric acid and citric acid is used as an effervescent base rather than either acid alone because when tartaric acid is used alone, chalky friable granules are produced and citric acid alone results in sticky mixture to difficult to granulate. The weakly acidic drugs when formulated in effervescent granular forms exhibit increased absorption from gastric environment as most of the drug remains in unionized form. The bitter taste of the drug can be masked by the effervescence that was produced when the formulation is mixed with water. Effervescent granules of weakly acidic drug, metronidazole were formulated by melt granulation process in which low melting binder composition comprising of a polymer and a hydrophobic meltable binder like PEG 4000 and cetyl alcohol is used. Formulations having varying composition of effervescents and meltable binder composition were prepared and evaluation tests for carr's index, effervescence cessation time, in-vitro drug release studies were performed. From the observations, the formulation having $5 \%$ meltable binder, cetyl alcohol was found to have excellent flow properties, $100 \%$ drug release and high effervesce cessation time.
\end{abstract}

INTRODUCTION: Effervescent granules are the granular dosage forms having drug and effervescent base ${ }^{1}$ which is composed of sodium bicarbonate, citric acid and tartaric acid, when added to water, the acids and the base react to liberate $\mathrm{CO}_{2}$, resulting in effervescence. Effervescent granules form attractive dosage form as the carbonated solution masks the undesirable taste of the drug. The weakly acidic drugs like metronidazole when formulated in effervescent granular forms exhibit increased absorption from gastric environment as most of the drug remains in unionized form.

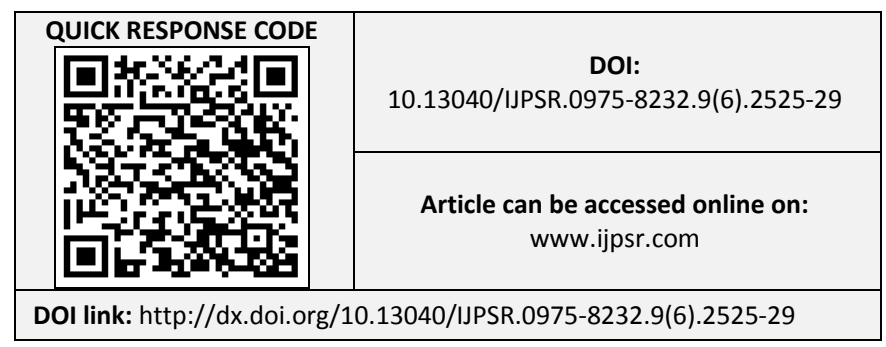

The effervescence released when the formulation is added to water masks the unpalatable taste of metronidazole. In the present study of effervescent granule formulation, melt granulation technique is employed for granulation. In a melt granulation process, the binder solution of a standard wet granulation process is replaced with a meltable binder. This binder can be added in molten form, but the high shear process offers the benefit of allowing the binder to be added in its solid state. Melting is achieved by the energy added through the mixer friction and the heated jacket of the bowl.

A low melting binder composed of a polymer and a hydrophobic meltable binder is used in the present work. The binder volume, binder rheology, binder surface property, and binder particle size on melt agglomeration influences formulation and process variables. The growth of melt agglomerate is promoted predominantly by an increase in 
viscosity, tack and specific volume as well as a decrease in surface tension of the molten binding liquid. The viscosity, tack, specific volume and surface tension govern the intra agglomerate mobility of molten binding liquid and state of liquid saturation of melt agglomerates. The influences of viscosity, tack and surface tension of molten binding liquid on melt agglomeration are affected by product temperature, mixing speed, and physicochemical properties of the fine solid particles. Widely used meltable binders in pharmaceutical industry include poloxamer 188, polyethylene glycol 2000 (PEG 2000), PEG 3000, PEG 4000, PEG 6000, gelucire 50/13 which have a range of melting point.

\section{MATERIALS AND METHODS:}

Materials: Metronidazole was procured from local vendor, Citric acid (Arrow Fine Chemicals, Rajkot, India), Tartaric acid (A. B. Enterprises, Mumbai, India), Fumaric acid (Alpha Chemika, Maharastra, India), Sodium bicarbonate (M R Scientific Suppliers, Pune, India), Cetyl alcohol (Alpha Chemika, Maharastra, India), PEG 4000 (Arrow Fine Chemicals, Rajkot, India) were procured and used in the investigation.

Formulation of Metronidazole Effervescent Granules: Metronidazole effervescent granules are prepared by melt granulation technique ${ }^{2,3}$. Melt granulation is also known as "Thermoplastic Granulation" as the granulation is achieved by adding a meltable binder which is in solid state at room temperature but preferably melts in the temperature range of $50{ }^{\circ} \mathrm{C}-80^{\circ} \mathrm{C}$.

No further addition of liquid binder or water is required in the process as the binder in the molten state itself act as granulating liquid and dried granules can be easily obtained by simple cooling at room temperature. Formulations were done using meltable binder (PEG 4000) and cetyl alcohol of concentrations $5 \%, 7.5 \%$ and $10 \%$ each by melt granulation technique. Formulations having different proportions of meltable binders ${ }^{4}$ were tabulated in Table 1.

Formulation of effervescent granules of metronidazole using different proportions of meltable binders is as follows:

- Weigh the required quantity of ingredients.
- Pass all the ingredients through sieve no.18.

- Add binder to the above ingredients.

- Heat it at temperature of about $50{ }^{0} \mathrm{C}$ to $80{ }^{0} \mathrm{C}$ till a molten mass is formed.

- Cool down the molten mass to room temperature.

- Pass the molten mass through sieve no. 8 or sieve no. 10 to obtain granules.

- Dry the granules at temperature not more than $60{ }^{\circ} \mathrm{C}$.

- Repeat the same process using different concentrations of binders $(5 \%, 7.5 \% \& 10 \%)$.

\section{Evaluation of Metronidazole Effervescent Granules: 5, 6,7}

Angle of Repose: The formulated effervescent granules were evaluated for their flow properties by measuring angle of repose. Angle of repose was determined by fixed funnel method. In this method, a funnel was secured with its tip at a given height (h), above a graph paper that is placed on a flat horizontal surface.

The blend was carefully poured through the funnel until the apex of the conical pile just touches the tip of the funnel. The radius of the base of the conical pile was measured. The angle of repose was calculated using the formula,

$\operatorname{Tan} \theta=\mathrm{h} / \mathrm{r}$

Where, $\theta=$ Angle of repose, $\mathrm{h}=$ Height of the cone, $r=$ Radius of the cone.

Carr's Index (\%): The Compressibility index (Carr's index) is a measure of the propensity of a powder to be compressed. It is determined from the bulk and tapped densities. In theory, the less compressible a material, the more flowable it is. As such, it measures the relative importance of interparticulate interactions. In a free flowing powder, such interactions are generally less significant and the bulk densities and tapped densities will be closer in value.

For poorer flowing materials, there are frequently greater inter-particle interactions and a greater difference between bulk and tapped densities will be observed. 
These differences are reflected in the Carr's Index ${ }^{8}$ which is calculated using the following formulae:

Compressibility index $=\left[\left(\rho_{\text {tap }}-\rho_{\mathrm{b}}\right) / \rho_{\text {tap }}\right] / \times 100$

Where, $\rho_{b}=$ Bulk Density, $\rho_{\text {tap }}=$ Tapped Density.

The standard chart of angle of repose and carr's index showing flow properties is given in the Table 2.

Effervescence Cessation Time: $100 \mathrm{ml}$ of distilled water was taken in $250 \mathrm{ml}$ beaker, one dose of effervescent granules was introduced in to the beaker, effervescence and effervescence cessation time were observed.

Standard Calibration Graph of Metronidazole: 20, 40, 60, 80 and $100 \mu \mathrm{g} / \mathrm{mL}$ concentrations of drug solutions were scanned against $0.1 \mathrm{~N} \mathrm{HCl}$ as reference solution at $277 \mathrm{~nm}$ under UV spectrophotometer. These working dilutions were scanned at $277 \mathrm{~nm}$ for their absorbencies by using UV spectrophotometer. A graph was plotted by taking absorbancies on Y-axis and concentration $(\mu \mathrm{g} / \mathrm{mL})$ on $\mathrm{X}$-axis. This graph yields standard calibration graph of drug solutions.

In-vitro Dissolution Studies: The effervescent granules were placed inside the dissolution vessel. The dissolution apparatus used for this study was USP TYPE II apparatus ${ }^{9}$ of which paddle was set at a speed of $75 \mathrm{rpm}$ Samples of $1 \mathrm{ml}$ were withdrawn at time intervals 10, 20, 30, 40, 50 and $60 \mathrm{~min}$. The volume of dissolution fluid is adjusted to $900 \mathrm{ml}$ by replacing $1 \mathrm{ml}$ of fresh dissolution medium after each sampling and thus sink condition was maintained. In this study the dissolution medium used was $0.1 \mathrm{~N} \mathrm{HCl}$ and temperature of $37 \pm 0.5{ }^{0} \mathrm{C}$ was maintained throughout the dissolution studies.

The release studies were conducted with 3 doses of effervescent granules and the mean values were plotted versus time. Each sample was diluted to 10 $\mathrm{ml}$ and analyzed at $277 \mathrm{~nm}$ using double beam UV and visible Spectrophotometer against reagent blank.

RESULTS AND DISCUSSION: In the present work, attempt was made to formulate and evaluate effervescent granules. Attempts were made to improve formulation technique by using meltable binders and also improve effervescence cessation time and drug release characteristics. Different compositions of meltable binder affect effervescence cessation time and drug release rate. The aim of this study is to enhance drug release rate and effervescence cessation time, thereby masking the bitter taste of drug and the drug of higher doses like metronidazole can be easily administered in the form of effervescent granules. Effervescent granules ${ }^{10}$ of metronidazole were developed using different proportions of meltable binders and the formulations having good flow properties were selected and evaluated. Different proportions of meltable binders used in formulations were tabulated in the Table 1.

Angle of repose: The formulations were evaluated for angle of repose by fixed funnel method and the following data was obtained for the formulations F1 to F7 and the flow properties ${ }^{11}$ can be determined by comparing with the standard data in the Table 2. Angle of repose and the flow property of the formulations were tabulated in the Table 3 .

Effervescence Cessation Time: Effervescence cessation time was determined in $100 \mathrm{~mL}$ of distilled water. The effervescence cessation time for the formulations F1 to F7 was tabulated in the Table 4.

Standard Calibration Graph of Metronidazole (in 0.1N $\mathbf{H C l}$ ): A graph was plotted by taking absorbancies of drug solutions on $\mathrm{Y}$-axis and concentration $(\mu \mathrm{g} / \mathrm{mL})$ on $\mathrm{X}$-axis. This graph yields standard calibration graph of drug solutions. The standard calibration data was given in Table 5 and graphical representation was given in Fig. 1.

TABLE 1: COMPOSITION OF EFFERVESCENT GRANULES

\begin{tabular}{ccccccccc}
\hline S. no. & Ingredients & $\mathbf{F}_{\mathbf{1}}$ & $\mathbf{F}_{\mathbf{2}}$ & $\mathbf{F}_{\mathbf{3}}$ & $\mathbf{F}_{\mathbf{4}}$ & $\mathbf{F}_{\mathbf{5}}$ & $\mathbf{F}_{\mathbf{6}}$ & $\mathbf{F}_{\mathbf{7}}$ \\
\hline 1 & Sodium bicarbonate & 2.050 & 2.050 & 2.050 & 2.050 & 2.050 & 2.050 & 2.050 \\
2 & Citric acid & 0.520 & 0.520 & 0.520 & 0.520 & 0.520 & 0.520 & 0.520 \\
3 & Tartaric acid & 0.152 & 0.152 & 0.152 & 0.152 & 0.152 & 0.152 & 0.152 \\
4 & Fumaric acid & 0.152 & 0.152 & 0.152 & 0.152 & 0.152 & 0.152 & 0.152 \\
5 & Metronidazole & 0.325 & 0.325 & 0.325 & 0.325 & 0.325 & 0.325 & 0.325 \\
6 & PEG 4000 & $5 \%$ & $7.5 \%$ & $10 \%$ & - & - & - \\
7 & Cetyl alcohol & - & - & - & $5 \%$ & $7.5 \%$ & $10 \%$ \\
\hline
\end{tabular}


TABLE 2: STANDARD CHART OF ANGLE OF REPOSE SHOWING FLOW PROPERTIES

\begin{tabular}{ccc}
\hline Angle of repose & Carr's index & Type of flow \\
\hline$<25^{0}$ & $5-15 \%$ & Excellent \\
$25-30^{0}$ & $12-16 \%$ & Good \\
$30-40^{0}$ & $18-21 \%$ & Fair to passable \\
- & $23-35 \%$ & Poor \\
- & $33-38 \%$ & Very poor \\
$>40^{0}$ & $>40 \%$ & Extremely poor \\
\hline
\end{tabular}

TABLE 3: DETERMINATION OF ANGLE OF REPOSE

\begin{tabular}{ccc}
\hline Formulation code & Angle of repose & Flow property \\
\hline F1 & $23^{0} .7^{1}$ & Excellent \\
F2 & $66^{0} .5^{1}$ & Extremely poor \\
F3 & $28^{0} .3^{1}$ & Good \\
F4 & $24^{0} .7^{1}$ & Excellent \\
F5 & $17^{0} .22^{1}$ & Excellent \\
F6 & $15^{0} .1^{1}$ & Excellent \\
F7 & $18^{0} .2^{1}$ & Excellent \\
\hline
\end{tabular}

From the data it was found that formulation F4 has excellent flow property

TABLE 4: DETERMINATION OF EFFERVESCENCE CESSATION TIME

\begin{tabular}{cc}
\hline Formulation code & Effervescence cessation time (sec) \\
\hline F1 & 501 \\
F2 & 436 \\
F3 & 239 \\
F4 & 625 \\
F5 & 337 \\
F6 & 145 \\
F7 & 141 \\
\hline
\end{tabular}

From the data formulation, F4 was found to show longer effervescence cessation time.

TABLE 5: STANDARD CALIBRATION GRAPH OF METRONIDAZOLE (IN 0.1 N HCI)

\begin{tabular}{ccc}
\hline S. no. & Concentration $(\boldsymbol{\mu g} / \mathbf{M I})$ & Absorbance \\
\hline 1 & 0 & 0 \\
2 & 10 & 0.126 \\
3 & 20 & 0.217 \\
4 & 40 & 0.450 \\
5 & 60 & 0.710 \\
6 & 80 & 0.927 \\
7 & 100 & 1.125 \\
\hline
\end{tabular}

TABLE 5: IN-VITRO DISSOLUTION STUDY FOR THE FORMULATIONS F1 TO F7

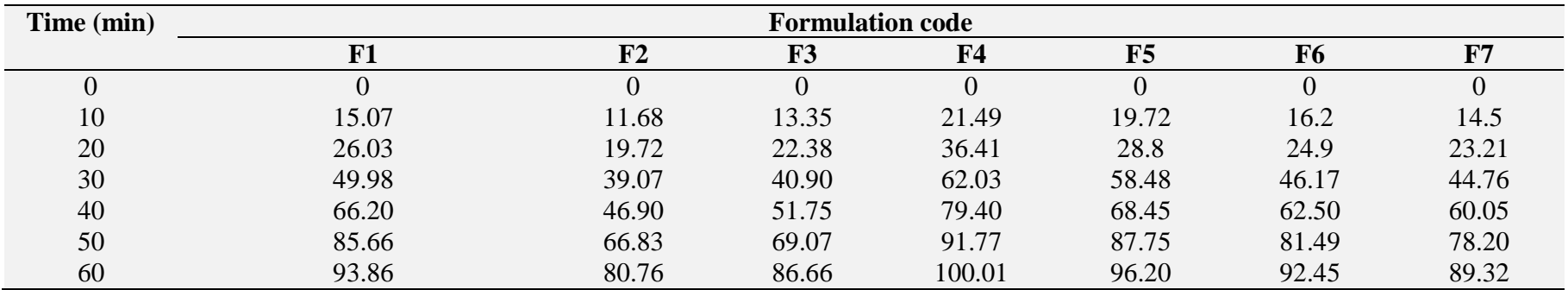

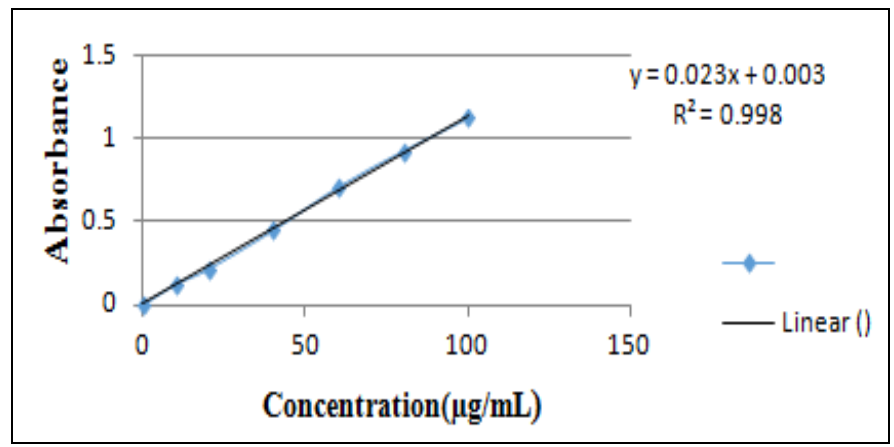

FIG. 1: STANDARD CALIBRATION GRAPH OF METRONIDAZOLE (IN 0.1N HCI)

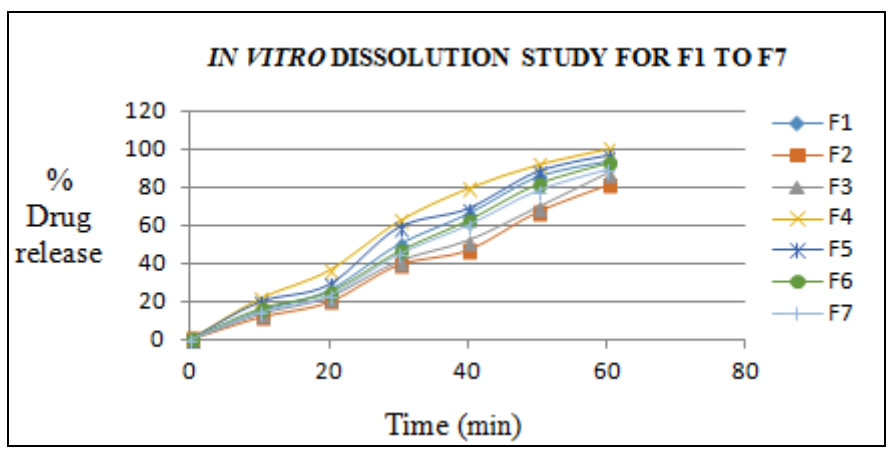

FIG. 2: IN-VITRO DISSOLUTION STUDY OF FORMULATIONS FROM F1 TO F7 
In-vitro Dissolution Studies: The formulations were evaluated for the in-vitro drug release in USP TYPE II dissolution apparatus using $0.1 \mathrm{~N} \mathrm{HCl}$ as dissolution medium. From the in-vitro dissolution studies, cumulative \% drug release was determined and was tabulated in Table 6 and graphically represented in Fig. 2.

In-vitro dissolution study was carried out and cumulative drug release was found for the formulations F1 to F7. From the data it was found that formulation F4, was found to release most of the drug at the end of 60 minute time interval.

CONCLUSION: Development of metronidazole, a weekly acidic drug in the form of effervescent granules not only masks the bitter taste of drug but also increases the bioavailabity of the drug by increasing the gastric absorption of the drug ${ }^{12}$. Metronidazole effervescent granules were formulated by melt granulation technique using meltable binders, PEG 4000 and cetylalcohol of varying concentrations.

Metronidazole effervescent granules of $5 \%$ cetylalcohol shows excellent flow properties and $100 \%$ drug release with high effervescent cessation time. Various formulations from $\mathrm{F} 1$ to $\mathrm{F} 7$ were developed and of which F4 was found to be optimized.

\section{ACKNOWLEDGEMENT: Nil}

\section{CONFLICTS OF INTEREST: Nil}

\section{REFERENCES:}

1. Odeku OA: Potentials of tropical starches as pharmaceutical excipients: A review. Starch/Stärke, 2013; 65(1-2): 89-106.

2. Halle Pradeep D: A review on melt granulation technique. Journal of Pharmacy and Phytotheraputics 2013; 1(3): 6 10.

3. Agrawal R and Yadav N: Pharmaceutical processing. A review on wet granulation technology. IJPFR 2011; 1(1): 65-83.

4. Prabhakar $\mathrm{C}$ and Krishna KB: A review on effervescent tablets. Int J Pharm Technol. 2011; 3: 704-12.

5. Moghimipour E, Akhgari $A$ and Ghassemian $Z$ : Formulation of glucosamine effervescent granules. Sci Med J. 2010; 9(1): 21-34.

6. Solanki HK, Basuri T, Thakkar JH and Patel CA: Recent advances in granulation technology. International Journal of Pharmaceutical Sciences Review and Research 2010; 5(3):4 8-54.

7. Banker GS and Anderson NR: Tablets: Lachman L, Lieberman $\mathrm{H}$, The theory and practice of Industrial Pharmacy. CBS publishers, New Delhi, 2009; 293-345.

8. Peter D: Oral solid dosage forms: Gibson M. Pharmaceutical preformulation and formulation. A practical guide from candidate drug selection to commercial dosage form, Interpharm/CRC, New York, 2008; 379-432.

9. Raymond $\mathrm{M}$ : Effervescent tablets: Lieberman HA, Lachman L and Schwartz TB: Pharmaceutical dosage forms, Marcel Dekker, Inc, New York, 2008; 2(1): 285328.

10. Cantor S and Gerhardt A: Pharmaceutical granulation processes: Mechanism and the use of binders; Pharmaceutical Dosage Forms: Tablets, 2008; 261-301.

11. Lim HP: Particle and powder bed properties: Pharmaceutical Dosage Forms - Tablets, 2008; 17-73.

12. Twitchell A: Mixing. In: Aulton ME, editor. Pharmaceutics: The Science of Dosage Form Design. New York: Churchill Livingstone; Edition $3^{\text {rd }} 2007$; 181-96.

How to cite this article:

Diyya ASM and Thomas NY: Formulation and evaluation of metronidazole effervescent granules. Int J Pharm Sci Res 2018; 9(6): 2525-29. doi: 10.13040/IJPSR.0975-8232.9(6).2525-29.

All @ 2013 are reserved by International Journal of Pharmaceutical Sciences and Research. This Journal licensed under a Creative Commons Attribution-NonCommercial-ShareAlike 3.0 Unported License.

This article can be downloaded to ANDROID OS based mobile. Scan QR Code using Code/Bar Scanner from your mobile. (Scanners are available on Google Playstore) 